\title{
T-cell Responses in Oiled Guillemots and Swans in a Rehabilitation Setting
}

\author{
Gera M. Troisi
}

Received: 6 July 2012/ Accepted: 24 February 2013/Published online: 20 March 2013

(C) Springer Science+Business Media New York 2013

\begin{abstract}
Aquatic birds are commonly affected by oil spills. Despite rehabilitation efforts, the majority of rehabilitated common guillemots (Uria aalge) do not survive, whereas mute swans (Cygnus olor) tend to have higher postrelease survival. Polyaromatic hydrocarbons (PAHs) present in crude oil and diesel are immunotoxic in birds affecting cell-mediated responses to immunogens. Because it is a target of PAH toxicity, T-lymphocyte response to controlled mitogen administration (phytohemagglutinnin test) was investigated in a scoping study as a potentially useful minimally invasive in vivo test of cell-mediated immunity. The test was performed on 69 mute swans and 31 common guillemots stranded on the Norfolk and Lincolnshire coastline and inland waterways in England (UK) either due to injury or to contamination with crude or diesel oil. T-lymphocyte response was significantly decreased in swans with greater oil scores. T-lymphocyte responses were also decreased in guillemots, but this finding was not statistically significant.
\end{abstract}

Stranded aquatic birds are a common feature after oil and diesel spill events. Birds become externally contaminated on contact with oil at the water's surface and ingest significant quantities of oil by preening resulting in plasma and tissue contamination with polycyclic aromatic hydrocarbons (PAHs) (Troisi \& Borjesson 2005; Troisi et al. 2006). There are considerable efforts to recover, clean, and rehabilitate oiled aquatic birds for release to the wild. In the United Kingdom, post-release survival of rehabilitated

G. M. Troisi $(\bowtie)$

School of Engineering and Design, Brunel University, Kingston Lane, Uxbridge, Middlesex UB8 3PH, UK

e-mail: gera.troisi@brunel.ac.uk oiled common guillemots (Uria aalge) is low, whereas survival of mute swans (Cygnus olor) contaminated mainly by localised incidental diesel spills in marinas and inland waterways tends to be much greater (Wernham et al. 1997). This is because guillemots are more sensitive to captivity and handling stress in rehabilitation than larger more robust species, such as swans. In addition, guillemots are usually oiled on contact with expansive areas of spilt crude oil in open sea or coastal areas leading to severe oiling of the plumage and subsequent oral exposure from preening (Grantham 2004). In contrast, swans are usually oiled on contact with less toxic and less viscous diesel oil from smaller localised spills in inland waterways. Beyond the immediate influence of the acute effects of oiling (weight loss, hypothermia), there are long-term chronic effects of PAH contamination, such as reproductive toxicity, thyroid disruption, hemolytic anaemia, and hepatic and renal toxicity, all of which pose further threats to the long-term survivability of aquatic birds that survive initial oiling (Peakall et al. 1981; Troisi et al. 2007; Leighton et al. 1983; Leighton 1993).

Postrelease survival of rehabilitated oiled birds in the wild is partly dependent on their resistance to pathogens, which may be compromised by PAH immunotoxicity (Briggs et al. 1996). Controlled dosing of gulls and ducks with crude oil have shown that there is a shift away from granulopoiesis to erythropoiesis to combat PAH-induced hemolytic anemia leading to decreased resistance to infection and splenic and bursal atrophy (Holmes et al. 1978; Rocke et al. 1984; Leighton 1986). Cell-mediated immunity is the main target for PAH immunotoxicity, whereas humoral immunity (antibody production) is less significantly affected (Rocke et al. 1984). Captivity and handling stress also influence immune competence in rehabilitated oiled aquatic birds potentially exacerbating $\mathrm{PAH}$-associated 
immunosuppression (Newman et al. 2000). The immediate impact of immune suppression on rehabilitated birds is decreased resistance to infections, which spread quickly in indoor environments where large numbers of birds are in close proximity, particularly after a large oil spill. For example, Aspergillosis spp. infections of the lung are frequently reported, and infection of injuries, such as hock lesions, which develop from standing on unsuitable substrates. Furthermore, inflammation of the intestinal mucosa due to oil ingestion also results in nutrient malabsorption resulting in weight loss, diarrhoea, and decreased intestinal T-lymphocyte production, thus allowing greater pathogen entry across the gut, which also influences survival (Newman et al. 2000; Briggs et al. 1996).

Biomarkers are useful tools for assessing exposure and effects of pollutants in wildlife. They constitute a biological response(s) that is dose-dependent with toxicant exposure. Biomarkers can be used to monitor pollutant exposure and/or effects and are intrinsically linked to the toxic mechanism by which they cause effects. For a biological response(s) to be a suitable biomarker for monitoring PAH exposure and associated PAH-induced health effects in live oiled wildlife, it must be measurable in samples collected nondestructively (e.g., blood). Candidate biomarkers must also be sufficiently specific and sensitive to monitor environmental exposures and induced by a known toxic mechanism. Tests evaluating changes in immune tissue/organ histopathology, plasma thyroid hormone, retinol, cell-mediated (T-cell response, differential leucocyte counts), and humoral (immunoglobulin concentrations) responses provide information on how contaminants interfere with immune function, and they have been used in avian biomonitoring studies (Peakall et al. 1981; Grasman et al. 1996; Newman et al. 2000; Smits et al. 2002; Troisi et al. submitted). The phytohemagglutinin (PHA) skin test is a simple nondestructive in vivo method to study how T-lymphocyte response to mitogenic challenge may be altered due to contaminant exposure (Grasman 2002). Indeed this test has been validated by controlled dosing studies with polychlorinated biphenyls (PCBs) in birds and has been successfully applied in biomonitoring studies of organochlorine exposure in wild bird populations (Grasman et al. 1996; Smits et al. 2002; Sagerup et al. 2009).

Humoral responses have been found in both biomonitoring and dosing studies to be less useful biomarkers of immunotoxicity because the primary target of immunotoxic pollutants (PAHs and PCBs) is cell-mediated immunity. Furthermore, immunoglobulin concentrations are more susceptible to confounding factors (e.g., inflammation, dehydration, nutritional status, age, and sex) (Grasman et al. 1996; Briggs et al. 1996). Nondestructive measurement of humoral immune response requires species-specific immunoglobulin assays, which are not yet commercially-available for birds. However, the PHA test has potential to be a useful cost-effective, nondestructive tool for assessing cell-mediated (T lymphocyte) immune competence in a rehabilitation setting. To investigate the stranded/injured mute swans and common guillemots admitted to East Winch Royal Society for Prevention of Cruelty to Animals (RSPCA) Wildlife Centre, many of which had been exposed to crude or diesel oils, they were subjected to PHA testing as part of a scoping study to establish reliability and practicality issues with using the test on smaller and larger species of oiled birds in a rehabilitation setting.

\section{Methods}

Stranded adult common guillemots ( $U$. aalge $)$ and adult mute swans $(C$. olor) admitted to the East Winch Wildlife Hospital RSPCA were PHA tested by veterinary staff within routine treatment under a United Kingdom Home Office Licence (sampling and treatment of live animals for their veterinary care). The guillemots were stranded along the Norfolk or Lincolnshire coastline as a result of injury or contamination with crude oil from the Tricolour spill in The French Channel (Grantham 2004), whereas swans were stranded in marinas and inland waterways in the local area from injuries or diesel contamination. Stranded birds admitted to the hospital for various reasons (e.g., injury, oiling, infection) during a 4-week period between December and January. It was not possible or ethical to obtain birds from the wild specifically for this study. It was not possible to achieve equal or adequate representation for each level of oil exposure, body condition, outcome (killed or released), age, or sex. The uncontaminated birds in this study were admitted due to physical trauma/injury.

All birds were in various states of stress, dehydration, and emaciation. Body weights were recorded on admission. Overall body condition was scored on the basis of availability of fat reserves on an arbitrary basis by eye as follows: $0=$ emaciated, $1=$ lean, $2=$ fair, and $3=$ good. External oiling of plumage was also scored on an arbitrary scale by eye as follows; $0=$ unoiled, $1=$ lightly oiled $(<50 \%$ coverage), $2=$ moderately oiled $50-75 \%$ coverage), and $3=$ heavily oiled ( $<75 \%$ coverage). Information on sex of the swans was not collected by the RSPCA and thus was not available to this study. Some of the birds that died were subject to postmortem examination to determine sex and age class (guillemots only) and to investigate the cause of death. The majority of the guillemots were killed due to the severe effects (including haemolytic anemia, muscle wasting, renal dysfunction) of worsening emaciation and cachexia $(N=15)$. A low number died or were killed due to physical injury $(n=2)$ or conditions, such as hock lesions $(n=2)$ and Aspergillosis spp. infections $(n=4)$ contracted well after 
admission as a result of being held in captivity, which is a commonly observed phenomenon in oiled bird rehabilitation centres (Balseiro et al. 2005).

To test T-lymphocyte response, the PHA test was performed on live guillemots and swans once they had been washed and stabilised after admission (1-3 days from admission; any birds with infection were excluded from the study). This involved the injection of mitogen $(100 \mu \mathrm{g}$ of PHA dissolved in sterile $0.1 \mathrm{ml}$ of Hartmann's solution) into the right foot web to generate an immune response and $0.1 \mathrm{ml}$ of sterile Hartmann's solution into the left foot web as a control. Quadruplicate measurements of foot web thickness at the injection site were undertaken with digital callipers (accuracy $\pm 0.01 \mathrm{~mm}$ ) immediately before injection and at $24 \mathrm{~h}$. The mean change in foot web thickness (in $\mathrm{mm}$ ) from the time of injection and after $24 \mathrm{~h}$ was calculated as PHA response by subtracting any change in foot web thickness observed in respective controls. Normally the patagium (wing web) is used for PHA injection in birds in an area from which feathers have been removed (Grasman 2002). However, most of the birds in this study were in a distressed condition, so veterinary staff decided it was more appropriate to inject the foot web (interdigital skin), which is more accessible, thereby minimising handling and further distress. This approach has been used successfully to assess immune function of juvenile chickens exposed to PCBs due to their small body size (Lavoie et al. 2007). It was not possible to test healthy unoiled guillemots in this study due to the restrictions of the United Kingdom Home Office Licence, under which the RSPCA staff were operating, which prohibits wild animals being sampled unless they require veterinary care.

\section{Results}

PHA cell responses were decreased by threefold in the most contaminated swans $($ score $=3$ ) compared with unoiled swans (Fig. 1). Paired comparison of mean data for each oil score category showed statistically significant decreases in response according to two-tailed student $t$ test as follows: 0 versus 1 ( $p<0.05), 0$ versus $2(p<0.01), 0$ versus $3(p<0.001), 1$ versus $3(p<0.01)$, and 2 versus 3 $(p<0.01)$ with the exception of scores 1 versus 2 . In guillemots, PHA response was decreased in heavily oiled birds ( score $=3$ ) compared with unoiled birds ( score $=0$ ), but differences between category means were not statistically significant (Fig. 1). Body weight and PHA response in swans and guillemots according to sex, age class, body condition, and outcome are listed in Table 1. Although not statistically significant, PHA response was less pronounced in female compared with male birds (age classes pooled). PHA responses were similar between age classes for both
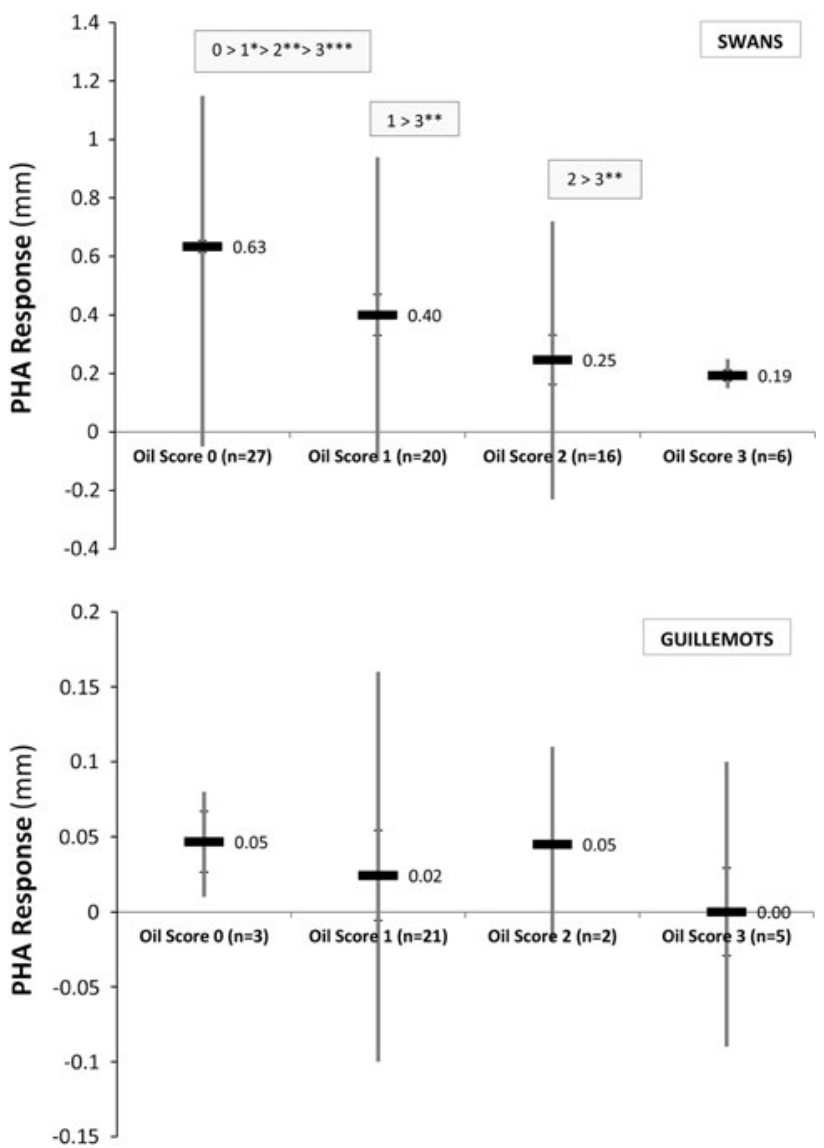

Fig. 1 Relationship between exposure and PHA response in mute swans (upper panel) and common guillemots (lower panel). The PHA response was calculated as change in mean foot web thickness minus change in control foot and are expressed here as means ( \pm SEs) and ranges. Where present, statistically significant differences between categories are indicated $\left({ }^{*} p<0.05 ; * * p<0.01 ; * * * p<0.001\right)$

species (sexes pooled). There were no significant differences in PHA response in guillemots on the basis of outcome and comparison of PHA response between outcomes could not be made because all swans survived. Swans with body condition score $=2$ had significantly greater body weights than birds with score $=1(p<0.001)$; no swans represented categories 0 and 3 . Although not statistically significant, body weights of guillemots with body condition score $=1$ were also heavier than those with poorer body condition scores; no guillemots represented categories 2 and 3. In Fig. 2, body weight and PHA response from both species are shown to be positively associated, but the correlations were not statistically significant.

\section{Discussion}

The PHA test was found to be a reliable method for measuring $\mathrm{T}$ cell-mediated responses in the foot web of 
Table 1 Species, age class and outcome related means ( \pm SDs) for body weight and T-cell response (change in foot web thickness)

\begin{tabular}{|c|c|c|}
\hline \multirow[t]{2}{*}{ Outcomes } & \multicolumn{2}{|l|}{ Mean \pm SD (range) } \\
\hline & Body weight (kg) & T-cell response (mm) \\
\hline Mute swans $(n=69)$ & $6.79 \pm 0.46(3.60-10.2)$ & $0.44 \pm 0.07(-0.23$ to 1.15$)$ \\
\hline $\operatorname{Sex}$ & $\mathrm{N} / \mathrm{D}$ & N/D \\
\hline \multicolumn{3}{|l|}{ Age class } \\
\hline Adult & $\begin{array}{l}8.07 \pm 0.60(5.70-12.10) \\
(n=15)\end{array}$ & $\begin{array}{l}0.39 \pm 0.06(-0.23 \text { to } 1.11) \\
(n=34)\end{array}$ \\
\hline Juvenile & $\begin{array}{l}6.13 \pm 0.82(3.60-7.90) \\
(n=6)\end{array}$ & $\begin{array}{l}0.42 \pm 0.08(0.01-1.10) \\
(n=14)\end{array}$ \\
\hline \multicolumn{3}{|l|}{ Body condition (score) } \\
\hline 0 & $(n=0)$ & $(n=0)$ \\
\hline 1 & $5.98 \pm 0.31(3.60-6.90)(n=13)$ & $0.37 \pm 0.06(-0.10$ to 1.11$)(n=31)$ \\
\hline 2 & $10.00 \pm 1.21(7.90-12.10)(n=4)$ & $0.32 \pm 0.12(-0.23$ to 0.94$)(n=12)$ \\
\hline 3 & $(n=0)$ & $(n=0)$ \\
\hline Outcome & N/A & N/A \\
\hline Common guillemots $(n=31)$ & $0.73 \pm 0.04(0.60-0.86)$ & $0.02 \pm 0.01(-0.10$ to 0.16$)$ \\
\hline \multicolumn{3}{|l|}{ Sex } \\
\hline Male $(n=20)$ & $0.74 \pm 0.02(0.61-0.86)$ & $0.03 \pm 0.01(-0.10$ to 0.16$)$ \\
\hline Female $(n=11)$ & $0.71 \pm 0.03(0.60-0.83)$ & $0.01 \pm 0.02(-0.09$ to 0.11$)$ \\
\hline \multicolumn{3}{|l|}{ Age class } \\
\hline Adult $(n=21)$ & $0.77 \pm 0.02(0.61-0.86)$ & $0.02 \pm 0.01(-0.10$ to 0.13$)$ \\
\hline Juvenile $(n=10)$ & $0.64 \pm 0.01(0.60-0.71)$ & $0.02 \pm 0.02(-0.01$ to 0.16$)$ \\
\hline \multicolumn{3}{|l|}{ Body condition (score) } \\
\hline $0(n=3)$ & $0.66 \pm 0.28(0.31-0.71)$ & $-0.02 \pm 0.02(-0.05$ to 0.02$)$ \\
\hline $1(n=28)$ & $0.74 \pm 0.16(0.60-0.86)$ & $-0.02 \pm 0.01(-0.16$ to 0.10$)$ \\
\hline $2(n=0)$ & - & - \\
\hline $3(n=0)$ & - & - \\
\hline \multicolumn{3}{|l|}{ Outcome } \\
\hline Dead $(n=28)$ & $0.72 \pm 0.18(0.60-0.86)$ & $0.03 \pm 0.01(-0.10$ to 0.16$)$ \\
\hline Released $(n=3)$ & $0.77 \pm 0.28(0.72-0.81)$ & $0.05 \pm 0.04(-0.02$ to 0.11$)$ \\
\hline
\end{tabular}

Outcome: N/A not applicable (as all were released), N/D not data available

oiled swans. The significantly lower level of swelling response measured in the foot web of heavily oiled swans was indicative of an immunosuppressed response. A reasonable explanation for this is the immunotoxicity exerted by the PAHs to which the birds were exposed. That the PHA foot web test was also successful for assessing immune-competence of guillemots in this study, but there was lack of a statistically significant association between oil exposure and PHA response mainly caused by insufficient representation of each oil score category, thus causing confounding factors to have great influence. The results of the PHA test were also compromised to some degree by the extreme level of emaciation and dehydration in the guillemots, which lead to poor circulation and cold legs and feet. Poor vascular circulation may have decreased T-cell delivery to the target site of PHA injection thereby influencing changes in foot web thickness. This problem this posed for testing was overcome by warming the bird's legs to boost poor circulation and soften the skin, which enabled reproducible injection and measurement. However, the exacerbated condition of the guillemots would no doubt have suppressed the PHA response. This is also the reasoning behind selecting the foot web skin as the PHA injection site instead of the wing web because it is better suited for smaller birds, such as chicks, as well as smaller species, which are more easily stressed by handling (Lavoie et al. 2007). Most of the guillemots in this study did not respond to treatment and died ( 28 of 31 birds) within a few months of admission due to their level of emaciation. Unfortunately, "normal T-lymphocyte" responses to PHA from healthy guillemots are not available in the published literature to provide control data, and it was not possible 

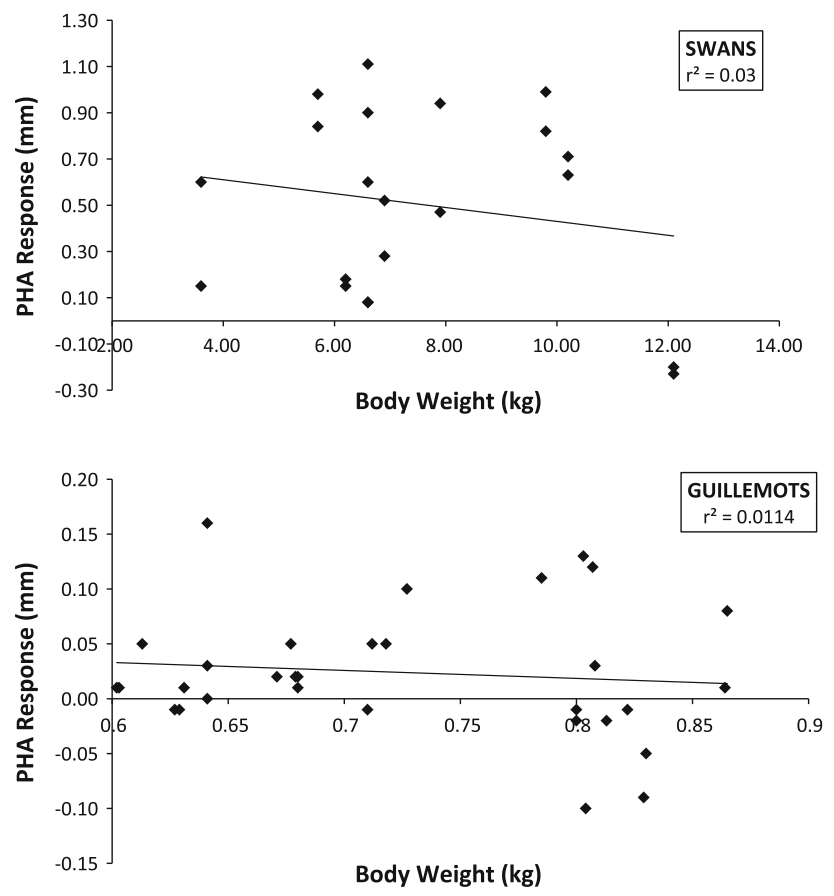

Fig. 2 Relationship between body weight and PHA response in mute swans and common guillemots. $n s$ not significant

under the United Kingdom Home Office Licence, under which the RSPCA was operating, to capture healthy guillemots or swans to serve as controls for this study.

The only comparable study of avian cell-mediated responses after exposure to petroleum chemicals is the controlled dosing of zebra finch chicks (Taeniopygia guttata) to consolidated oil sands tailing (CT) water, where the lack of any immunosuppressive effects was proposed to be due to the low PAH concentrations found in CT water $(<2 \mu \mathrm{g} / \mathrm{l}$; Madill et al. 2001) and short duration of exposure (Smits and Williams 1999). This in contrast with what the immunosuppressive effects observed in this study. An explanation for this is the different exposure scenarios. Birds in this study were exposed to comparatively greater concentrations of PAH by preening crude or diesel oil from their feathers during a period of days or weeks, and petroleum oils contain significantly greater quantities of PAHs than CT water (Madill et al. 2001). Suppression of PHA responses associated with organic contaminant exposure observed in this study was in agreement with PCB-induced suppression observed in Great Lake caspian terns (Sterna caspia) and herring gulls (Larus argentatus), in which a $30-45 \%$ decrease in responses was observed in heavily contaminated populations (Grasman et al. 1996). Furthermore, in controlled PCB dosing studies with American kestrels (Falco sparverius), PHA responses were also found to be significantly decreased (Smits et al. 2002).

T-cell response in both swans and guillemots was generally greater in individuals with higher body condition scores, but differences between scores were not significant owing to insufficient representation of each category. It has already been established that body mass is positively correlated with T-cell response in unexposed birds (AlonsoAlvarez and Tella 2001). The lack of any relationship between body mass or body condition score and PHA responses in swans may strengthen the possibility that oil exposure was the predominating factor influencing $\mathrm{T}$-cell response in this study. However, because starvation decreases peripheral T-lymphocyte activity and inflammatory response, it is plausible that the emaciated condition apparent in most of the guillemots suppressed their T-cell responses. Indeed the immunosuppressive effect of emaciation will likely influence the recovery of free-living oiled seabirds in the wild because emaciation is the predominant immediate impact of oiling (Wanless et al. 2000). In the case of age- or sex-dependent differences in PHA responses, no statistically significant differences were observed in guillemots due to small sample size and lack of complete age and sex data in this study. It was not possible to examine the influence of age and sex on PHA response in swans because age and sex data were not recorded. It is important to undertake further studies with larger sample sizes to investigate whether juvenile and adult female birds, particularly juvenile female birds, are more sensitive to the immunosuppressive effects of PAHs than other members of the population due to their importance to breeding and population recovery. In terms of differences in PHA response with outcome (released or died), no conclusions could be made because of the lack of representation from each outcome category (the majority of the guillemots died, and all of the swans were released). The greater survivorship of swans compared with guillemots can be explained as follows. First, swans were exposed to diesel oil, not crude oil, as were the guillemots, which is less toxic (contains a lower proportion of PAHs). In addition, being slightly more water soluble, diesel oil is easier to successfully wash off the plumage. Due to the difference in exposure and the fact that swans are a larger, more robust species, oiled swans respond much better than guillemots to rehabilitation.

Although the PHA test provided an indication of the responsive capacity of cell-mediated immunity targeted toward a specific mitogen, the PHA test is not able to assess the integrity of the functionality of these cells. Despite this, the PHA test was shown in this study to be useful for costeffective and minimally-invasive means of assessment of cell-mediated immunity in a rehabilitation setting. One of the roles of well-differentiated T-cells is to seek and destroy antigens, and this study provides some evidence that oil exposure may decrease T-lymphocyte immune response. However, further validation is necessary, including the testing of healthy control birds, to qualify the 
dose-response relationship of PHA response to confirm its suitability as a biomarker of oil-induced PAH immunotoxicity. In the rehabilitation context, handling and captivity stress may have influence, albeit minor, on PHA response compared with the influence of infections, such as Aspergillosis spp. and hock infections, which can occur in captivity (Balseiro et al. 2005). Therefore, PHA testing is best applied to stabilised rehabilitated guillemots that have regained their weight as a means to establish whether individuals are adequately immune-competent before release to improve their chances of survival. In addition, by this time, rehabilitated birds would have acclimatised to handling and captivity, so the influence of handling stress on the PHA test results should be minimal.

\section{Conclusion}

PAHs affect cell-mediated immunity to a greater degree than humoral immunity (Rocke et al. 1984), and the PHA test has been successfully used to assess the immunotoxicity of pollutants on T-lymphocyte responses in various bird species (Grasman 2002). The PHA test is cost-effective, simple to use, and minimally invasive, and this scoping study showed that by boosting leg blood circulation and using the foot web as the PHA injection site, the PHA test can provide reliable immune competence assessment of even smaller stressed and emaciated oiled seabirds, thus suiting the requirements of wildlife rehabilitation centres. Although this was only a preliminary investigation, the PHA test was used in this scoping study to measure T-lymphocyte response in stranded swans and guillemots exposed to varying degrees with diesel and crude oils in a wildlife rehabilitation centre. The study found that immune-competence of swans was significantly decreased in birds with greater oil scores, but no such conclusion could be drawn with guillemots due to the influence of various confounding factors (emaciation, dehydration, stress) and inadequate numbers of control (unexposed) birds in this study. The impacts of severe emaciation and dehydration from oiling on this sensitive species make it more difficult to measure small changes in tissue inflammation as a biological response. Consequently, the PHA test is best suited to test guillemots with good body condition, for example, to confirm T-lymphocyte integrity before release in rehabilitated birds. The overall influence of oil-induced immunosuppression on the survivability of rehabilitated oiled birds is not known. At the population level, aquatic birds face numerous ecological and toxicological challenges ranging from oil spill disasters, food chain contamination (organic and heavy-metal pollutants), overfishing, pathogenic challenges, and climate change, to mention a few. A subtle change in cell-mediated immune competence due to $\mathrm{PAH}$ exposure may exacerbate these impacts and is worthy of further study to improve rehabilitation practice and survivability.

\section{References}

Alonso-Alvarez C, Tella JL (2001) Effects of environmental food restriction and body mass changes on the avian T-cell mediated immune response. Can J Zool 79:101-105

Balseiro A et al (2005) Pathological features in marine birds affected by the prestige's oil spill in the north of Spain. J Wildl Dis 41(2):371-378

Briggs KT, Yoshida SH, Gershwin ME (1996) The influence of petrochemicals and stress on the immune system of seabirds. Reg Toxicol Pharmacol 2:145-155

Grantham M (2004) Age structure and origins of British and Irish guillemots (Uria aalge) recovered in recent European oil spills. Atl Seabirds 6(3):95-108

Grasman KA (2002) Assessing immunological function in toxicological studies of avian wildlife. Integr Comp Biol 42:34-42

Grasman KA et al (1996) Organochlorine-associated immunosuppression in pre-fledgling caspian terns and herring gulls from the Great Lakes: an eco-epidemiological study. Environ Health Perspect 104(Suppl 4):829-842

Holmes WN, Cronshaw J, Gorsline J (1978) Some effects of ingested petroleum on seawater-adapted ducks (Anas platyrhyncos). Environ Res 17:177-190

Lavoie ET, Wiley F et al (2007) Effect of in ovo exposure to an organochlorine mixture extracted from double crested cormorant eggs and PCB 126 on immune function of juvenile chickens. Arch Environ Contam Toxicol 53:655-661

Leighton FA (1986) Conical, gross and histological findings in herring gulls and Atlantic puffins that Ingested Purdhoe Bay Oil. Vet Pathol 23:254-263

Leighton FA (1993) The toxicity of petroleum oils to birds. Environ Rev 1:92-103

Leighton FA et al (1983) Heinz-body anemia from the ingestion of crude oil: primary toxic effect in marine birds. Science 220:871873

Madill REA et al (2001) Madill preliminary risk assessment of the wet landscape option for reclamation of oil sands mine tailings: bioassays with mature fine tailings pore water. Environ Toxicol 16(3):197-208

Newman SH et al (2000) An experimental soft-release of oil-spill rehabilitated American Coots (Fulica americana): II. Effects on health and blood parameters. Environ Pollut 107:295-304

Peakall DB et al (1981) Endocrine dysfunction in seabirds by ingested oil. Environ Res 24:6-14

Rocke TE et al (1984) Oil and related toxicant effects on mallard immune defences. Environ Res 33:343-352

Sagerup KA et al (2009) The toxic effects of multiple persistent organic pollutant exposures on the post-hatch immunity maturation of glaucous gulls. J Toxicol Environ Health A 72:870-883

Smits JE, Williams TD (1999) Validation of immunotoxicology technique in passerine chicks exposed to sands tailing water. Ecotoxicol Environ Safety 44:105-112

Smits JE et al (2002) Thyroid hormone suppression and cell-mediated immunomodulation in American kestrel (Falco sparverius) exposure to PCBs. Arch Environ Contam Toxicol 43:338-344

Troisi GM (submitted) Association between thyroid-stimulating hormone (TSH) and polyaromatic hydrocarbon (PAH) exposure in oiled guillemots. Int J Environ Res 
Troisi GM, Borjesson L (2005) Development of an immunoassay for the determination of polyaromatic hydrocarbons PAHs in plasma samples from oiled sea birds. Environ Sci Technol 38:3748-3755

Troisi GM et al (2006) Polyaromatic hydrocarbon and PAH metabolite burdens in oiled common guillemots (Uria aalge) stranded on the East Coast of England (2001-2002). Environ Sci Technol 40:7938-7944

Troisi GM et al (2007) Biomarkers of polycyclic aromatic hydrocarbon (PAH)-associated hemolytic anemia in oiled wildlife. Environ Res 105:324-329
Wanless S, Harris MP et al (2000) Changes in body mass of common guillemots Uria aalge in south-east Scotland throughout the year: implications for the release of cleaned birds. Ringing Migr 20:134-142

Wernham CV, et al. (1997) Survival rates of rehabilitated guillemots. British Trust for Ornithology, BTO Research Report, No. 186 